\title{
TRICRITICAL ISING MODELS AND THE SMOOTHNESS POSTULATE*
}

\author{
F. Harbus and H. E. Stanley \\ Massachusetts Institute of Technology, Cambridge, Mass. 02139
}

\section{ABSTRACT}

As applied to magnetic systems with tricritical points, the smoothness postulate predicts constant critical-point exponents along the second order portion of the phase boundary. We consider here the behavior of the critical-point exponent $\gamma_{\text {st }}$ characterizing the staggered susceptibility $X_{s t} \sim\left[T-T_{c}(H)\right]^{-\gamma_{s t}}$ in two Ising model antiferromagnets with tricritical points. The evidence indicates that $\gamma_{s t}$ remains $5 / 4$ for a wide range of fields, and appears to rule out a continuously changing index along the critical line.

In contrast to ferromagnets, antiferromagnets display a phase transition which persists even in the presence of an external field H. The field serves to oppose the intrinsic antiferromagnetic ordering of the system, resulting in a line of critical points in the $\mathrm{H}-\mathrm{T}$ plane $\mathrm{T}=\mathrm{T}_{\mathrm{c}}(\mathrm{H})$, with the critical temperature a decreasing function of $H$. One of the consequences of the "smoothness" postulate posed by Griffiths 1 is that the critical indices characterizing the phase transition will not change as one moves down along the critical line, at least for small critical fields near the Néel point.

In metamagnetic systems, however, it is observed that for low enough temperatures, the nature of the phase transition does in fact change drastically--the external field induces a first order rather than second order transition to the paramagnetic phase. Griffiths $^{2}$ called the point of changeover from second to first order behavior the "tricritical point" (TCP), and went on to suggest that such a point is a likely possibility for the breakdown of smoothness. 1 The TCP is the terminus of three critical lines and represents a special symmetry point in the metamagnetic phase diagram spanned by $H, T$, and a staggered magnetic field $\mathrm{H}_{\mathrm{St}}$. Some reports of TCP exponents have been made for both real and model magnetic systems. $3-5$

Rapaport and Domb 6 applied series extrapolation techniques to test the smoothness predictions for a 2-sublattice Ising model antiferromagnet with nearest neighbor (negative) exchange only, uniform with respect to lattice direction. The phase transition in such a "simple" antiferromagnet is expected to remain second order all the way to absolute zero. To study models with tricritical behavior, we have applied high-temperature series expansions to two different Ising model antiferromagnets which incorporate ferro*Work supported by NSF, ONR, and AFOSR. 
magnetic interactions within each sublattice. In the context of these models, the smoothness postulate predicts that exponents will stay the same along the second order portion of the phase boundary, and change discontinuously at the TCP. Of course, close to the TCP crossover effects between the critical line exponents and the TCP exponents will occur. Once close enough to the critical line, however, the critical line exponents are predicted to dominate.

Our calculated phase boundaries for the two Ising tricritical models and estimates of their respective TCP susceptibility exponents are given elsewhere. ${ }^{5}$ In this paper we focus upon the behavior of the exponent $\gamma_{s t}$ characterizing the divergence of the staggered susceptibility $\chi_{\text {st }}$ along the critical line,

$$
x_{\text {st }} \sim\left[\mathrm{T}-\mathrm{T}_{\mathrm{c}}(\mathrm{H})\right]^{-\gamma} \text { st }
$$

Our evidence over a wide range of field values $H$ supports the smoothness prediction that $Y_{\text {st }}$ is independent of $H$. Although it was impossible to verify this arbitrarily close to the estimated TCP due to increasing irregularity of the series, we believe that our results cover a sufficiently wide range of fields to argue convincingly against a continuous variation of $\gamma_{s t}$ along the critical line.

It is not surprising that closer to the TCP a finite number of series terms will begin to fail to reveal true asymptotic critical behavior. A closely analogous, and conceptually simpler, situation occurs in the application of series to study critical behavior when a three-dimensional lattice crosses over to a two-dimensional lattice as the exchange parameter linking adjacent layers goes to zero.?

The first tricritical Ising model treated has a Hamiltonian

$$
\mathcal{H C}=-J_{x y} \sum_{\langle i j\rangle}^{x y} s_{i} s_{j}-J \sum_{\langle i j\rangle}^{z} s_{i} s_{j}-\mu H \sum_{i} s_{i}
$$

where the first sum is over nearest neighbor (nn) spins coupled within an $\mathrm{x}-\mathrm{y}$ plane on the sc lattice, while the second sum is over $\mathrm{nn}$ spins coupled along the $\mathrm{z}$ direction. To simulate a metamagnet, we take $J_{x y}>0$ (ferromagnetic) and $J_{z}<0$ (antiferromagnetic). We generate high-temperature series expansions to eighth order in inverse temperature for the two-spin correlation function; these series are exact in the external field. Fig. 1 shows estimates of $\gamma_{s t}$ based upon ratio analysis methods applied to the series for $\chi_{s t}$ for the parameter choice $J_{x y}=1, J_{z}=-1$. Represented are " $1 / n^{1 "}$ sequences for several values of the variable $h=\mu \mathrm{H} / \mathrm{k}_{\mathrm{B}} \mathrm{T}$, the natural variable in which the field enters the expansions. The plots of Fig. 1 are actually determined from the series after a bilinear transformation on the original expansion variable is carried out in order to mitigate the effect of oscillations in the ratio plots. 


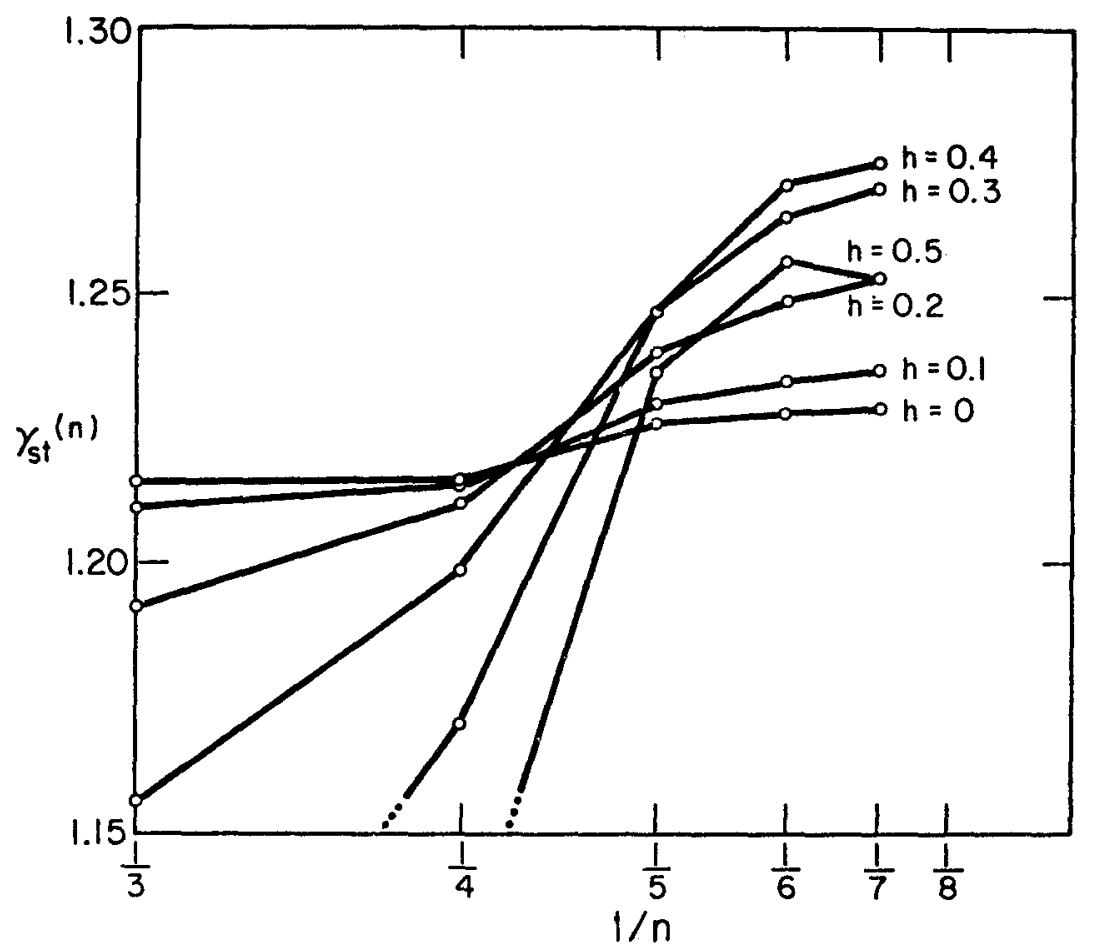

Fig. 1. Sequences of estimates $\gamma_{\text {st }}^{(n)}$ vs. 1/n for Hamiltonian (2) with $J_{x y}=1, J_{z}=-1$ along various $h \equiv \mu \mathrm{H} / \mathrm{k}_{\mathrm{B}} \mathrm{T}$ paths. The $\gamma_{\text {st }}(\mathrm{n})$ are calculated from ratio method on $\chi_{s t}$ series after transformation from expansion variable $\beta \equiv 1 / \mathrm{k}_{\mathrm{B}} \mathrm{T}$ to $\beta *=\beta /(1+1.5 \beta)$.

The estimates in Fig. 1 for $\gamma_{\text {st }}$ are closely scattered on both sides of the smoothness postulate prediction of $5 / 4$. The final two estimates for any $h$ path fall in the range of $1.250 \pm .025$. To gauge the extent of the fields covered in this plot, we note that the $h=.5$ path corresponds to a critical field of 1.7 (in dimensionless units), to be compared with the maximum $\mathrm{T}=0$ critical field of 2 . Adding to the evidence that the exponent $5 / 4$ indeed correctly characterizes the behavior of $X_{\text {st }}$ in this range is the Padé approximant analysis carried out on the same set of series. Each $X_{\text {st }}$ series is raised to the $4 / 5$ power and the dominant physical singularity located in the resulting Pade table. There is very good agreement between the critical temperatures given by the ratio method and those located by the Padé technique. Such consistency would not be expected if the $\chi_{\text {st }}$ series did not in fact diverge with a 5/4 power law.

The second tricritical model has nearest neighbor antiferromagnetic bonds and next nearest neighbor ferromagnetic bonds on the sc lattice. In an obvious notation, 


$$
\mathcal{C}=-J_{1} \sum_{\langle i j\rangle}^{n n} s_{i} s_{j}-J_{2} \underset{\langle i j\rangle}{\sum} s_{i j} s_{j}-\mu H \sum_{i} s_{i}
$$

with $J_{1}<0$ and $J_{2}>0$. Fig. 2 gives estimates for $\gamma_{\text {st }}$ for this model with $J_{1}=-1$ and $J_{2}=+1 / 2$. The sequences here are based upon a slightly modified version of the ratio method in which one forms sequences of linear extrapolants of an original set of estimates. As can be seen from the rather fine scale in Fig. 2, the final estimates for $\gamma_{\text {st }}$ are extremely close to 1.250 . There is some upward curvature, but this is present even in the $h=0$ sequence. In zero field the staggered susceptibility will have the same exponent as the uniform susceptibility in the corresponding ferromagnet, well established to be $5 / 4$ for the three dimensional Ising model.

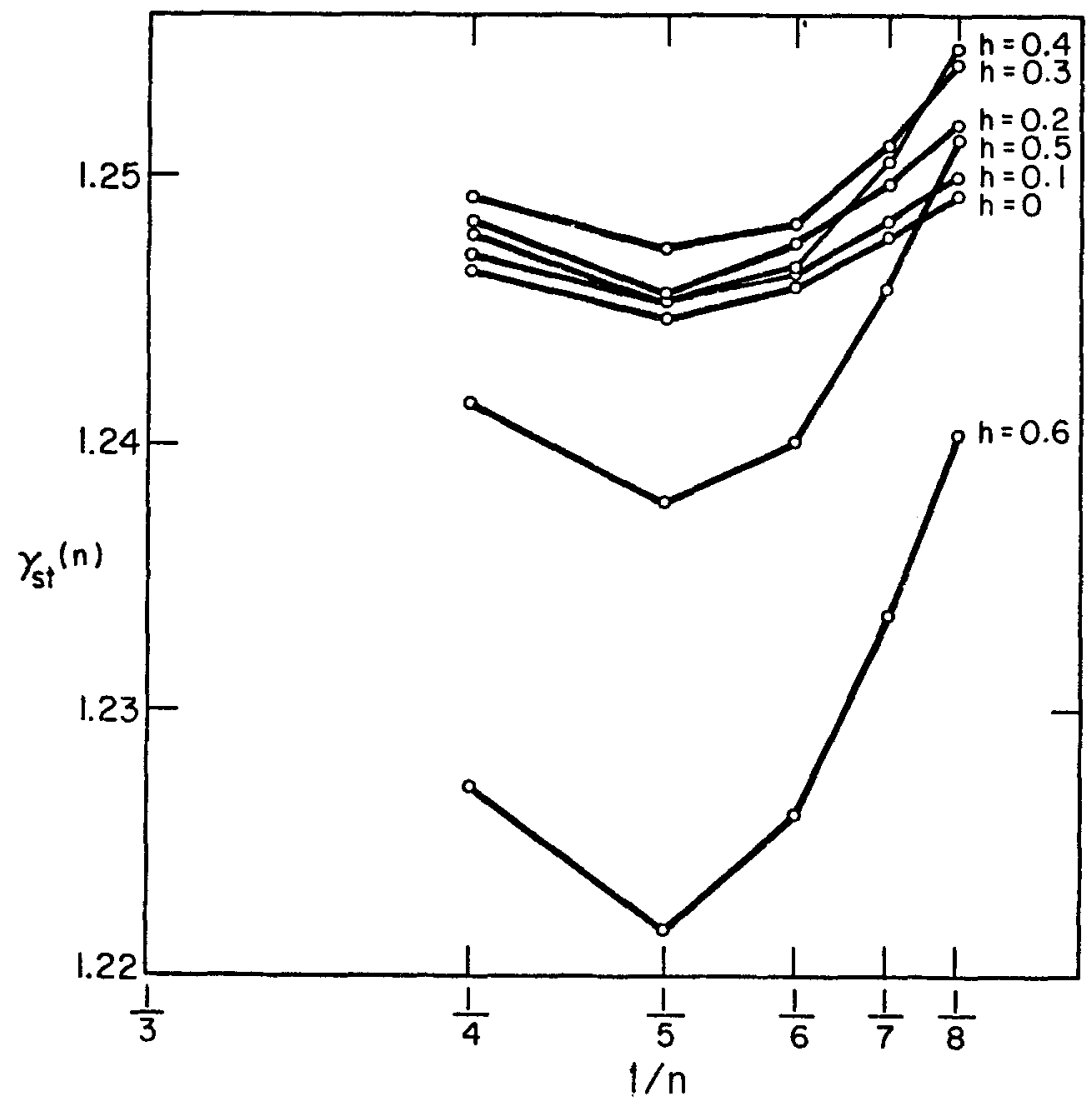

Fig. 2. Sequences of estimates $\gamma_{s t}^{(\mathrm{n})}$ vs. $1 / \mathrm{n}$ for Hamiltonian (3) with $\mathrm{J}_{1}=-1, \mathrm{~J}_{2}=+1 / 2$ along various $h$ paths. The $\gamma$, nt are a set of linearty extrapolated exponents. No bilinear transformation on the expansion variable $\beta$ is necessary. 
The plots certainly give little indication of $\gamma_{\text {st }}$ moving away from $5 / 4$ to any significant degree for the fields shown. In this model, the $T=0$ critical field is 6 , while the $h=.6$ path corresponds to critical field of 4.7. Again, consistency between ratio and Padé estimates for the critical temperatures in this range (and somewhat beyond) is excellent. Closer to the estimated TCP the ratios on the $X_{\text {st }}$ series give apparently decreasing values for $Y_{\text {st }}$. However, we believe this to be a spurious effect which would not be observed if sufficiently longer series were available, and conclude that our overall evidence supports the smoothness postulate prediction for both tricritical models.

\section{REFERENCES}

1. R.B. Griffiths, in Critical Phenomena in Alloys, Magnets, and Superconductors, edited by R. E. Mills, E. Ascher, and R. I. Jaffee (McGraw-Hill, New York, 1971), pp. 377-391.

2. R. B. Griffiths, Phys. Rev, Lett. 24, 715 (1970).

3. (a) D. P. Landau, B. E. Keen, B. Schneider, and W. P. Wolf, Phys. Rev. B3, 2310 (1971); (b) W. B.Wolf, B. Schneider, D. P. Landau, and B.E. Keen, Phys. Rev. B5, 4472 (1972).

4. D. P. Landau, Phys. Rev. Lett. 28, 449 (1972).

5. F. Harbus and H. E. Stanley, Phys. Rev. Lett. 29, 58 (1972); see also Proc. International Conference on Padé Approximants, Canterbury, England, July 1972, P.R. Graves Morris, Ed. (Academic Press, London, 1973).

6. D. C. Rapaport and C. Domb, J. Phys. C4ㅗ, 2684 (1971).

7. F. Harbus and H.E. Stanley, submitted for publication. 